\title{
ON THE TOTAL GRAPH OF A FINITE COMMUTATIVE RING
}

\author{
M. H. Shekarriz!, M. H. Shirdareh Haghighi, H. Sharif \\ Department of Mathematics, College of Science, \\ Shiraz University, 71454, Shiraz, Iran
}

\begin{abstract}
Let $R$ be a finite commutative ring with $1 \neq 0$. In this article, we study the total graph of $R$, denoted by $\tau(R)$, determine some of its basic graph-theoretical properties, determine when it is Eulerian, and find some conditions under which this graph is isomorphic to $\operatorname{Cay}(R, Z(R) \backslash\{0\})$. We shall also compute the domination number of $\tau(R)$.
\end{abstract}

Keywords: Cayley graphs, Total graph of a commutative ring, Zero-divisors

\section{INTRODUCTION}

Let $R$ be a commutative ring with $1 \neq 0, Z(R)$ its set of zero-divisors, and $R^{*}$ its set of units. Anderson and Badawi (2008) introduced the total graph of $R$, which we denote here by $\tau(R)$, as a simple graph with all elements of $R$ as vertices and for distinct $x, y \in R$, the vertices $x$ and $y$ are adjacent if and only if $x+y \in Z(R)$. They also studied some basic properties of $\tau(R)$ such as diameter, girth, connectivity, etc., mostly without the assumption that $R$ is finite. Akbari et al. (2009) have shown that if $R$ is a finite commutative ring and $\tau(R)$ is connected, it is also Hamiltonian. Maimani et al. have characterized all finite rings whose total graphs are planar or toroidal and have also shown that for every positive integer $g$, there are only finitely many finite rings (up to isomorphism) whose total graphs have genus $g$.

Remark 1.1. Anderson and Badawi (2008) use the notation $T(\Gamma(R))$ instead of $\tau(R)$. We prefer $\tau(R)$ because $T(\Gamma(R))$ has a different meaning in graph-theoretical context (see, for example, Behzad (1970)).

The definition of $\tau(R)$ may bring to mind the definition of the Cayley graph, $\operatorname{Cay}(R, Z(R) \backslash\{0\})$, which we denote here by $C(R)$. Hence, hereinafter, $C(R)$ represents the simple graph with vertex set $R$ and for distinct

\footnotetext{
${ }^{1}$ E-mail addresses: mhshekarriz@gmail.com (M. H. Shekarriz), shirdareh@susc.ac.ir (M. H. Shirdareh Haghighi), sharif@susc.ac.ir (H. Sharif).

2000 Mathematics Subject Classification: 13M99; 05C25; $05 \mathrm{C} 60$.
} 
$x, y \in R$, the vertices $x$ and $y$ are adjacent if and only if $x-y \in Z(R)$. Cayley graphs are widely studied in algebraic graph theory, and most of their graph-theoretical properties are known. For instance, it is known that $C(R)$ is a $(|Z(R)|-1)$-regular graph. For a general reference on algebraic graph theory, see Beineke and Wilson (2004). Meanwhile, Akhtar et al. (2009) have also studied the unitary Cayley graph of a finite $\operatorname{ring} R, \operatorname{Cay}\left(R, R^{*}\right)$, which is the complement of $C(R)$.

Besides the resemblance between the definitions of $\tau(R)$ and $C(R)$, they may be quite different in some of their graph-theoretical properties. In this article, we answer the naturally arising question: under what conditions on a finite commutative ring $R$, do we have $\tau(R) \simeq C(R)$ ?

We need some well-known facts about commutative rings: If $R$ is an Artinian ring, then either $R$ is local with its maximal ideal $\mathfrak{M}$, or $R \cong$ $R_{1} \oplus \cdots \oplus R_{k}$, where $k \geq 2$ and each $R_{i}$ is local with maximal ideal $\mathfrak{M}_{i}$; this decomposition is unique up to permutation of factors, see Atiyah and Macdonald (1969). Moreover, if $R$ is finite, then every element of $R$ is either a unit or a zero-divisor, i.e., $|Z(R)|=|R|-\left|R^{*}\right|$. Furthermore, if $R$ is also a local ring with maximal ideal $\mathfrak{M}$, then $\mathfrak{M}=Z(R)$, and there exists a prime $p$ such that the characteristic of the residue field $R / \mathfrak{M}$ is $p$, and $|R|,|\mathfrak{M}|$, and $|R / \mathfrak{M}|$ are all powers of $p$. Moreover, if $R \cong R_{1} \oplus \cdots \oplus R_{k}$, then $\left(u_{1}, \ldots, u_{k}\right)$ is a unit in $R$ if and only if $u_{i} \in R_{i}^{*}$ for each $i=1, \ldots, k$, and therefore we have $\left|R^{*}\right|=\left|R_{1}^{*}\right| \times \cdots \times\left|R_{k}^{*}\right|$.

In this article, we denote the residue field $R_{i} / \mathfrak{M}_{i}$ by $F_{i}$, the quotient map by $\pi_{i}: R_{i} \longrightarrow F_{i}$, and $\left|F_{i}\right|$ by $f_{i}$. As mentioned in Akhtar et al. (2009), after appropriate permutation of factors, we may assume that $f_{1} \leq \ldots \leq f_{k}$.

For the moment, suppose that $R$ is an infinite commutative ring. Then either $R$ has more than one zero-divisor, which means that it has infinitely many zero-divisors, or $R$ is an integral domain, which means that its total graph is the disjoint union of infinitely many $K_{1}$ 's or $K_{2}$ 's. In the former case, the degree of each vertex in $\tau(R)$ is infinite, which means that $\tau(R)$ is not locally finite and most of graph-theoretical properties such as being Eulerian or the domination number, etc. are meaningless or seem to be hard to determine. Thus, hereinafter, we assume that all rings are finite commutative with $1 \neq 0$. Meanwhile, we use the notations and definitions of graph theory from West (2000).

\section{BASIC PROPERTIES OF $\tau(R)$}

The following two propositions can be easily proved using theorems of Anderson and Badawi (2008, 2.2, 2.6, 3.3, 3.4 and 3.14): 
Proposition 2.1. Let $R$ be a finite commutative ring. Then $\tau(R)$ is connected if and only if $Z(R)$ is not an ideal of $R$, if and only if $R$ is not a local ring. Moreover, if $R$ is not a local ring, then $\operatorname{diam}(\tau(R))=2$, and $\operatorname{gr}(\tau(R))=3$ except when $R \cong \mathbb{Z}_{2} \oplus \mathbb{Z}_{2}$, where we have $\operatorname{gr}(R)=4$.

Proposition 2.2. Let $R$ be a local ring and $\beta=|R / Z(R)|$. Then

(a) if $2 \in Z(R)$, then $\tau(R)$ is the union of $\beta$ disjoint $K_{|Z(R)|}$ 's;

(b) if $2 \notin Z(R)$, then $\tau(R)$ is the disjoint union of one copy of $K_{|Z(R)|}$ and $(\beta-1) / 2$ copies of $K_{|Z(R)|,|Z(R)|}$.

Moreover, $\operatorname{gr}(\tau(R))=3$ if $|Z(R)| \geq 3$, otherwise $\operatorname{gr}(\tau(R))=\infty$.

Remark 2.3. Anderson and Badawi (2008, 2.6: (3)(b)) have stated that when $Z(R)$ is an ideal of $R$, then $\operatorname{gr}(\tau(R))=4$ if and only if $2 \notin Z(R)$ and $|Z(R)|=2$. But this case cannot happen since there are only two (finite) rings with $|Z(R)|=2$, say $\mathbb{Z}_{4}$ and $\mathbb{Z}_{2}[X] /\left(X^{2}\right)$, and in both of them $2 \in Z(R)$. (This Remark is also clear form Lemmas 2.5 and 2.6 below.)

In $\tau(R)$, the degree of 0 is $|Z(R)|-1$. Maimani et al. have proved a lemma similar to the following:

Lemma 2.4. Let $R$ be a finite commutative ring and $x$ be a vertex of $\tau(R)$. Then the degree of $x$ is $|Z(R)|$ if $x+x=2 x \notin Z(R)$, otherwise the degree of $x$ is $|Z(R)|-1$. In particular, $2 \in Z(R)$ if and only if $\tau(R)$ is a $(|Z(R)|-$ 1)-regular graph.

Proof. For every $z \in Z(R)$, there is a unique $a=z-x \in R$ such that $x+a=z$. Then $x$ is adjacent to $a$ unless $a=x$. If $2 x \in Z(R)$, then since $x$ cannot be adjacent to itself, $\operatorname{deg} x=|Z(R)|-1$. If $2 x \notin Z(R)$, then for every zero-divisor $z$ we have $z-x \neq x$, and hence $\operatorname{deg} x=|Z(R)|$. In particular, if $2 \in Z(R)$, then for every $x \in R$, we have $\operatorname{deg} x=|Z(R)|-1$. Therefore, $\tau(R)$ is a $(|Z(R)|-1)$-regular graph. Now suppose that $2 \notin Z(R)$. Then $\operatorname{deg} 1=|Z(R)| \neq|Z(R)|-1=\operatorname{deg} 0$, i.e., $\tau(R)$ is not a regular graph.

We also need the following two lemmas whose proofs are simple and are left to the reader.

Lemma 2.5. Let $R$ be a finite commutative ring. Then $2 \in Z(R)$ if and only if 2||$R \mid$.

Lemma 2.6. Let $R$ be a finite commutative ring. If $|R|$ is odd, then $\left|R^{*}\right|$ is even.

Summing up, we have the following theorem:

Theorem 2.7. Let $R$ be a finite commutative ring. Then

(a) if $|R|$ is even, then $\tau(R)$ is a $(|Z(R)|-1)$-regular graph, 
(b) if $|R|$ is odd and $r \in R^{*}$ (respectively, $r \in Z(R)$ ), then $\operatorname{deg} r=|Z(R)|$ (respectively, $\operatorname{deg} r=|Z(R)|-1)$.

Remark 2.8. Note that 1 and 0 are not adjacent in $\tau(R)$. Therefore, $\tau(R)$ is never a complete graph. Furthermore, we show that $\tau(R)$ is never an odd cycle either. To the contrary, suppose that $\tau(R)$ is an odd cycle. Hence $\operatorname{deg} 1=\operatorname{deg} 0=2=|Z(R)|-1$. Therefore, by Lemma 2.4 we have $2 \in Z(R)$, and hence by Lemma 2.5 , we must have 2||$R \mid$, which is a contradiction since $|R|$ is odd.

\section{$3 \quad$ WHEN IS $\tau(R)$ EULERIAN?}

A graph is said to be Eulerian if it has a closed trail containing all edges. It is well-known that a graph is Eulerian if and only if it is connected and its vertex degrees are all even, see West (2000).

Lemma 3.1. Let $R$ be a finite commutative ring. Then the following three conditions are necessary for $\tau(R)$ to be an Eulerian graph:

(a) $Z(R)$ is not an ideal of $R$,

(b) $|Z(R)|$ is an odd integer,

(c) $2 \in Z(R)$.

Proof. Condition (a) is necessary since otherwise by Proposition 2.1, $\tau(R)$ is not connected and cannot be Eulerian. (b) is also necessary since otherwise $\operatorname{deg} 0=|Z(R)|-1$ is odd, and thus $\tau(R)$ cannot be Eulerian. Condition (c) is also necessary, since otherwise by Lemma 2.4 we have $\operatorname{deg} 1=|Z(R)|=$ $1+\operatorname{deg} 0$, and hence $\tau(R)$ cannot be an Eulerian graph since it has at least one vertex with an odd degree.

Remark 3.2. The three conditions above are also sufficient because they imply that $\tau(R)$ is a connected regular graph of an even degree, i.e., $\tau(R)$ is Eulerian. Moreover, while conditions (a) and (b) hold, condition (c) above could be replaced by

$\left(c^{\prime}\right) \quad 1+1=0$, i.e., the characteristic of $R$ is 2 .

Condition $\left(\mathrm{c}^{\prime}\right)$ always implies (c). Conversely, assume necessary conditions (a) and (b) hold. By Lemma 2.5, we have $2 \in Z(R)$ implies 2||$R \mid$. Meanwhile, by condition (b), $|Z(R)|$ is odd and thus $\left|R^{*}\right|$ is also odd. Therefore, there exists some $u \in R^{*}$ such that $u=-u$. Then $u u^{-1}=-u u^{-1}$ which 
means that $1=-1$. Hence $2=0$.

Theorem 3.3. Let $R$ be a finite commutative ring. Then the graph $\tau(R)$ is Eulerian if and only if $R$ is isomorphic to a direct sum of two or more finite fields of even orders, i.e., $R \cong \bigoplus_{i=1}^{k} \mathbb{F}_{2^{t_{i}}}$ for some $k \geq 2$.

Proof. If $R \cong \bigoplus_{i=1}^{k} \mathbb{F}_{2^{t_{i}}}$ for some $k \geq 2$, then $\tau(R)$ is Eulerian by Lemma 3.1 and Remark 3.2. To prove the converse, suppose that $R$ is a ring whose total graph is Eulerian. Consequently, by Lemma 3.1, $R \cong R_{1} \oplus \cdots \oplus R_{k}$, $k \geq 2$. Meanwhile, by Remark 3.2 , we must have $1+1=0$, which means that $1_{R_{i}}+1_{R_{i}}=0$ for all $i=1, \ldots, k$. Hence, by Lemma 2.5 , it can be inferred that $\left|R_{i}\right|$ 's are all even. If $\left|Z\left(R_{i}\right)\right| \geq 2$ for some $i=1, \ldots, k$, then 2||$Z\left(R_{i}\right) \mid$, and hence $\left|R_{i}^{*}\right|=\left|R_{i}\right|-\left|Z\left(R_{i}\right)\right|$ is even. Therefore, $\left|R^{*}\right|=\left|R_{1}^{*}\right| \times \cdots \times\left|R_{k}^{*}\right|$ is also even, i.e., $|Z(R)|$ is even which is a contradiction because $\tau(R)$ is an Eulerian graph and by Lemma $3.1(\mathrm{~b}),|Z(R)|$ must be odd. Thus, we must have $\left|Z\left(R_{i}\right)\right|=1$ for all $i=1, \ldots, k$. Therefore, the $R_{i}$ 's are all finite fields of even orders, i.e., $R \cong \bigoplus_{i=1}^{k} \mathbb{F}_{2^{t_{i}}}$.

Example 3.4. $\tau\left(\mathbb{Z}_{2} \oplus \mathbb{Z}_{2}\right)$ and $\tau\left(\mathbb{Z}_{2} \oplus \mathbb{Z}_{2} \oplus \mathbb{Z}_{2}\right)$ are shown in figures 1 and 2, respectively. They are both Eulerian:

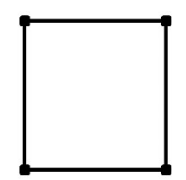

Figure 1. $\tau\left(\mathbb{Z}_{2} \oplus \mathbb{Z}_{2}\right)$.

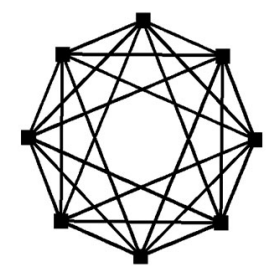

Figure 2. $\tau\left(\mathbb{Z}_{2} \oplus \mathbb{Z}_{2} \oplus \mathbb{Z}_{2}\right)$.

\section{DOMINATION NUMBER}

In a graph $G$, a set $S \subseteq V(G)$ is called a dominating set if every vertex not in $S$ has a neighbor in $S$. The domination number $\gamma(G)$ is the minimum size 
of a dominating set in $G$.

Theorem 4.1. Let $R$ be a finite commutative ring. We have $\gamma(\tau(R))=f_{1}$, except when $R$ is an integral domain of an odd order, where $\gamma(\tau(R))=\frac{f_{1}-1}{2}+$ 1 .

Proof. Let us treat the local case first. If $R$ is a local ring and $|R|$ is odd, then by Proposition 2.2, $\tau(R)$ is a disjoint union of one copy of $K_{|Z(R)|}$ and $\left(f_{1}-1\right) / 2$ copies of $K_{|Z(R)|,|Z(R)|}$. If besides we have $|Z(R)|=1$, then every dominating set must contain the vertex 0 and at least one vertex from each copy of $K_{1,1}$, and hence $\gamma(\tau(R))=\frac{\left(f_{1}-1\right)}{2}+1$. If $|R|$ is odd and $|Z(R)| \neq 1$, then every dominating set of a copy of $K_{|Z(R)|,|Z(R)|}$ must contain at least 2 vertices, one from each partite set. Therefore, we have $\gamma(\tau(R))=f_{1}$.

If $R$ is a local ring and $|R|$ is even, then $\tau(R)$ is a disjoint union of $f_{1}$ copies of $K_{|Z(R)|}$. Hence every dominating set requires at least one vertex from each copy. Thus $\gamma(\tau(R))=f_{1}$.

Now suppose that $R$ is not a local ring. Then $R \cong R_{1} \oplus \cdots \oplus R_{k} ; k \geq 2$. Choose $f_{1}$ elements $x_{j}=\left(x_{1 j}, \ldots, x_{k j}\right) \in R$, for $j=1, \ldots, f_{1}$, such that $\pi_{1}\left(x_{1 j}\right) \neq \pi_{1}\left(x_{1 j^{\prime}}\right)$ for $j \neq j^{\prime}$. Put $S=\left\{x_{j} \mid j=1, \ldots, f_{1}\right\}$, and suppose that $y=\left(y_{1}, \ldots, y_{k}\right)$ is an arbitrary element of $R \backslash S$. Then there is an $i=1, \ldots, f_{1}$ such that $\pi_{1}\left(x_{1 i}\right)=\pi_{1}\left(y_{1}\right)$. If $f_{1}$ is even, let $j=i$, else let $j$ be the index of the unique element of $S, x_{j}$ say, such that $\pi_{1}\left(x_{1 j}\right)=-\pi_{1}\left(x_{1 i}\right)$. Thus $y_{1}+x_{1 j} \in Z\left(R_{1}\right)$, and hence $y$ and $x_{j}$ are adjacent in $\tau(R)$. Therefore, $S$ is a dominating set and $\gamma(\tau(R)) \leq|S|=f_{1}$.

It remains to prove that $\gamma(\tau(R)) \geq f_{1}$. Let $A=\left\{a_{i}=\left(a_{1 i}, \ldots, a_{k i}\right) \in\right.$ $R \mid i=1, \ldots, t$ and $\left.t<f_{1}\right\}$. We show that $A$ is not a dominating set, and this completes the proof. For each $j=1, \ldots, k$, since $t<f_{1} \leq f_{j}$, there is $b_{j} \in R_{j}$ such that $\pi_{j}\left(b_{j}\right) \neq-\pi_{j}\left(a_{j i}\right)$ for each $i=1, \ldots, t$. Put $b=\left(b_{1}, \ldots, b_{k}\right)$. Then $b+a_{i} \notin Z(R)$ for each $i=1, \ldots, t$, which means that $b$ does not have a neighbor in $A$, and hence $A$ is not a dominating set.

Example 4.2. A dominating set for $\tau(R)$ for $R \cong \mathbb{Z}_{2}, \mathbb{Z}_{4}, \mathbb{Z}_{4} \oplus \mathbb{Z}_{3}$, or $\oplus_{i=1}^{k} \mathbb{Z}_{2}$ is $\left\{0_{R}, 1_{R}\right\}$. A dominating set for $\tau\left(\mathbb{Z}_{45}\right)$ is $\{\overline{0}, \overline{1}, \overline{2}\}$.

\section{$5 \quad$ WHEN $\tau(R) \simeq C(R) ?$}

In order to show that $\tau(R)$ and $C(R)$ are isomorphic, it is sufficient to find a bijection $f: R \longrightarrow R$ satisfying the following condition:

$$
a+b \in Z(R) \Longleftrightarrow f(a)-f(b) \in Z(R)
$$

Obviously, if $2 \notin Z(R)$, then $\tau(R) \nsucceq C(R)$ because by Lemma 2.4, $2 \notin$ $Z(R)$ implies that $\tau(R)$ is not a regular graph, while $C(R)$ is always regular. 
Hence $2 \in Z(R)$ is a necessary condition for the isomorphism $\tau(R) \simeq C(R)$. Meanwhile, if $R$ is a ring with characteristic 2, then $\tau(R) \simeq C(R)$; since $f=\mathrm{id}_{R}$ is the desired graph isomorphism because $x=-x$ for every $x \in R$, and

$$
a+b \in Z(R) \Longleftrightarrow f(a)-f(b)=a-b=a+b \in Z(R) .
$$

Moreover, if $R$ is a local ring (i.e., $Z(R)$ is an ideal of $R$ ) and $2 \in Z(R)$, then again $f=\mathrm{id}_{R}$ gives the isomorphism $\tau(R) \simeq C(R)$; since $a+b \in Z(R)$ implies $a+b-2 b \in Z(R)$, which means that $f(a)-f(b)=a-b \in Z(R)$.

In order to generalize the two previous cases, let $R \cong R_{1} \oplus \cdots \oplus R_{k}$ be a ring such that each $R_{i}$ is a local ring of even order. Thus by Lemma 2.5, we have $2_{R_{i}} \in Z\left(R_{i}\right)$ for each $i=1, \ldots, k$. In this case, if $a=\left(a_{1}, \ldots, a_{k}\right)$ and $b=\left(b_{1}, \ldots, b_{k}\right)$ are elements of $R$ and $a+b \in Z(R)$, then there exists an $i$ with $1 \leq i \leq k$ such that $a_{i}+b_{i} \in Z\left(R_{i}\right)$. Hence by the same reasoning, we have $a_{i}+b_{i}-2 b_{i}=a_{i}-b_{i} \in Z\left(R_{i}\right)$, which implies that $a-b \in Z(R)$. Therefore, if $R \cong R_{1} \oplus \cdots \oplus R_{k}$ with $k \geq 1$, and every $R_{i}$ is a local ring of even order, then $f=\mathrm{id}_{R}$ gives the isomorphism $\tau(R) \simeq C(R)$. The reader should verify that this case also covers the first case when $\operatorname{char}(R)=2$.

Now suppose that $R \cong R_{1} \oplus \cdots \oplus R_{k}$ such that every $R_{i}$ is local and $F_{1}=R_{1} / \mathfrak{M}_{1} \cong \mathbb{Z}_{2}$. Then if $u_{1}$ and $v_{1}$ are units in $R_{1}$, we have $u_{1}+v_{1} \in$ $\mathfrak{M}_{1}=Z\left(R_{1}\right)$. In this case, put

$$
A=\left\{\left(a_{1}, \ldots, a_{k}\right) \in R \mid a_{1} \in Z\left(R_{1}\right)\right\}
$$

and define $f: R \longrightarrow R$ by

$$
f(x)= \begin{cases}x & x \in A \\ -x & x \in A\end{cases}
$$

Let $a=\left(a_{1}, \ldots, a_{k}\right)$ and $b=\left(b_{1}, \ldots, b_{k}\right)$ be elements of $R$ such that $a+b \in$ $Z(R)$. Then there are three possibilities:

(i) $a, b \in A$; then $f(a)-f(b)=a-b=\left(a_{1}-b_{1}, \ldots, a_{k}-b_{k}\right)$ belongs to $Z(R)$ since $Z\left(R_{1}\right)$ is an ideal of $R_{1}$.

(ii) $a \in A$ and $b \notin A$ or vice versa; then $f(a)-f(b)=a+b$ which is already in $Z(R)$.

(iii) $a, b \notin A$; then $a_{1}, b_{1} \in R_{1}^{*}$, and since $F_{1}=R_{1} / \mathfrak{M}_{1} \cong \mathbb{Z}_{2}$, we have $-a_{1}+b_{1} \in Z\left(R_{1}\right)$. Hence in this case, we have $f(a)-f(b)=-a+b$ which is also in $Z(R)$.

Consequently, $f$ gives the desired isomorphism $\tau(R) \simeq C(R)$, since each possibility implies $f(a)-f(b) \in Z(R)$ and $f$ is a bijection. 
However, if $R \cong R_{1} \oplus \cdots \oplus R_{k}, 2 \in Z(R)$, but $F_{i}=\frac{R_{i}}{\mathfrak{M}_{i}} \not \mathbb{Z}_{2}$ for each $i=1, \ldots, k$, then there is no guarantee for the existence of an isomorphism $\tau(R) \simeq C(R)$.

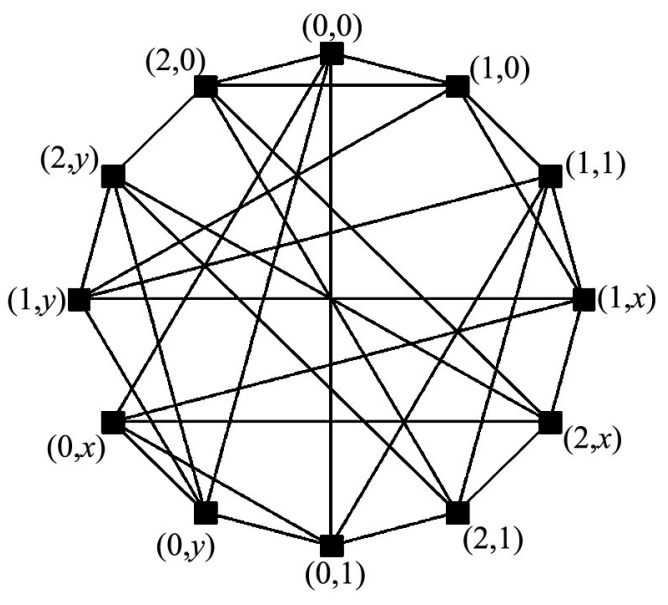

Figure 3. $C\left(\mathbb{Z}_{3} \oplus \mathbb{F}_{4}\right)$.
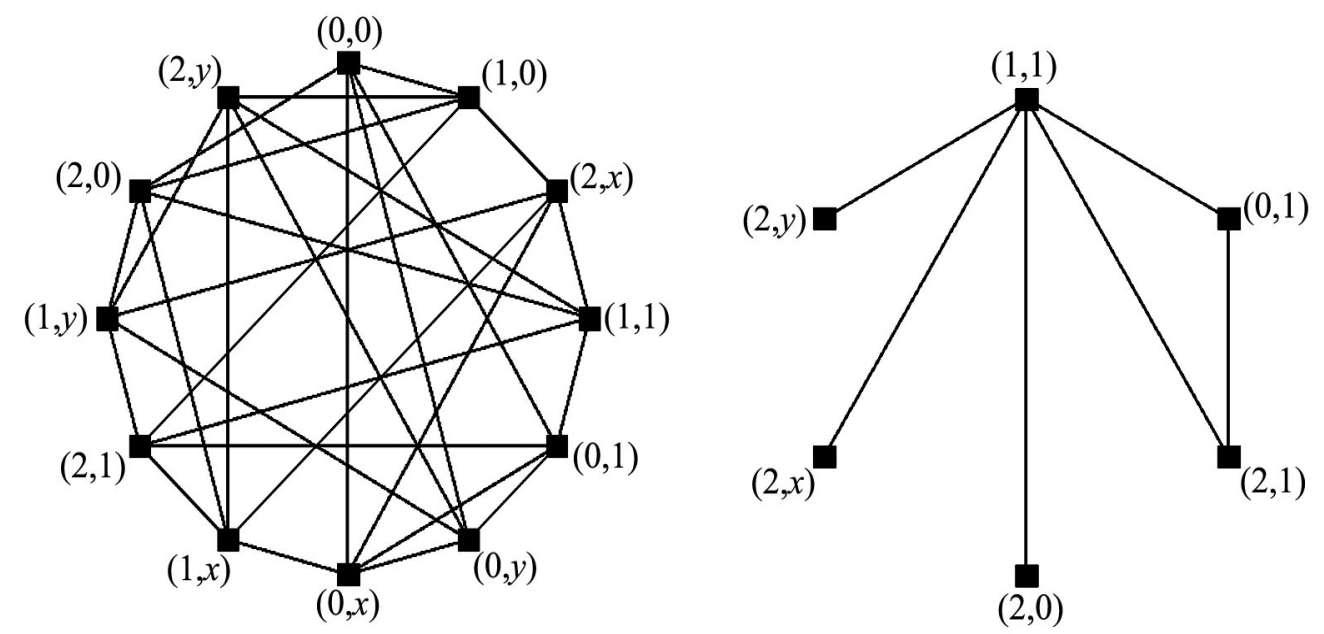

Figure 4. $\tau\left(\mathbb{Z}_{3} \oplus \mathbb{F}_{4}\right)$ and its induced subgraph $\tau\left(\mathbb{Z}_{3} \oplus \mathbb{F}_{4}\right)[N[(1,1)]]$.

Example 5.1. $\tau\left(\mathbb{Z}_{3} \oplus \mathbb{F}_{4}\right) \not \subset C\left(\mathbb{Z}_{3} \oplus \mathbb{F}_{4}\right)$. To see this, suppose $\mathbb{F}_{4}=$ $\{0,1, x, y\}$. Then the graph $C\left(\mathbb{Z}_{3} \oplus \mathbb{F}_{4}\right)$ has three disjoint 4-cliques (see Figure 3):

$$
\begin{aligned}
& \{(0,0),(0,1),(0, x),(0, y)\}, \\
& \{(1,0),(1,1),(1, x),(1, y)\}, \quad \text { and } \\
& \{(2,0),(2,1),(2, x),(2, y)\} .
\end{aligned}
$$


Hence every vertex in $C\left(\mathbb{Z}_{3} \oplus \mathbb{F}_{4}\right)$ belongs to a 4 -clique. But the vertex $(1,1)$ in $\tau\left(\mathbb{Z}_{3} \oplus \mathbb{F}_{4}\right)$ is not a vertex of a 4 -clique (see Figure 4 ). Therefore, $\tau\left(\mathbb{Z}_{3} \oplus \mathbb{F}_{4}\right) \not 千 C\left(\mathbb{Z}_{3} \oplus \mathbb{F}_{4}\right)$.

Theorem 5.2. Let $R$ be a finite commutative ring. Then the two graphs $\tau(R)$ and $C(R)$ are isomorphic if and only if at least one of the following conditions is true:

(a) $R \cong R_{1} \oplus \cdots \oplus R_{k}, k \geq 1$, and each $R_{i}$ is a local ring of an even order,

(b) $R \cong R_{1} \oplus \cdots \oplus R_{k}, k \geq 2$, and each $R_{i}$ is a local ring and $f_{1}=2$.

Proof. By the above discussion, if at least one of (a) or (b) is true, then $\tau(R) \simeq C(R)$. So, we only prove the converse. Suppose (a) and (b) do not hold for a ring $R$. If $2 \notin Z(R)$, then by the discussion at the beginning of this section, we have $\tau(R) \not C C(R)$. So, suppose that $2 \in Z(R)$. Therefore, $R \cong R_{1} \oplus \cdots \oplus R_{k} ; k \geq 2$ or else $R$ is local and $2 \in Z(R)$ implies that condition (a) is true. Furthermore, we have $R_{i} / Z\left(R_{i}\right) \neq \mathbb{Z}_{2}$ for all $i=1, \ldots, k$, but since $2 \in Z(R)$, it can be inferred that $R_{i} / Z\left(R_{i}\right) \cong \mathbb{F}_{2^{t}}$, for some $i=1, \ldots, k$ and $t \geq 2$. Moreover, there exists an $i, 1 \leq i \leq k$, such that $\left|R_{i}\right|$ is odd, because otherwise condition (a) is true again. So, we assume that $\left|R_{1}\right|, \ldots,\left|R_{j}\right|$ are all even and $\left|R_{j+1}\right|, \ldots,\left|R_{k}\right|$ are all odd, after appropriate permutation of factors of course.

In order to prove that $\tau(R)$ and $C(R)$ are not isomorphic, we use a method similar to the proof of Example 5.1. In fact, to prove that these two graphs are not isomorphic, we consider maximal cliques that contain an edge.

If $R_{i} / Z\left(R_{i}\right) \cong \mathbb{F}_{2^{t}}$ for some $t \geq 2$, then every vertex $a=\left(a_{1}, \ldots, a_{k}\right)$ belongs to a clique of maximal size $|R| / f_{i}$ in both graphs $\tau(R)$ and $C(R)$ because $\pi_{i}\left(a_{i}\right)=-\pi_{i}\left(a_{i}\right)$. But besides belonging to these distinct maximal cliques, for each $l=j+1, \ldots, k$, the vertex $a$ belongs to a maximal $\left(|R| / f_{l}\right)$-clique in $C(R)$. Because for each $b=\left(b_{1}, \ldots, b_{k}\right)$ and $c=\left(c_{1}, \ldots, c_{k}\right)$ which are adjacent to $a$, provided that $a_{l}-b_{l} \in Z\left(R_{l}\right)$ and $a_{l}-c_{l} \in Z\left(R_{l}\right)$, we have also $b_{l}-c_{l} \in Z\left(R_{l}\right)$. This means that $b$ and $c$ are also adjacent in $C(R)$. Therefore, if $b$ is adjacent to $a$, depending on the index $i$ that $a_{i}-b_{i} \in Z\left(R_{i}\right)$, we can say that $a$ and $b$ are both vertices of a maximal $\left(|R| / f_{i}\right)$-clique in $C(R)$. Therefore, every edge of $C(R)$ belongs to a maximal $\left(|R| / f_{i}\right)$-clique, for some $i=1, \ldots, k$.

Now, in $\tau(R)$, put $x=\left(0_{R_{1}}, \ldots, 0_{R_{j}},-1_{R_{j+1}}, \ldots,-1_{R_{k}}\right)$. Then $x$ is adjacent to $1=(1, \ldots, 1)$, and for all $i=1, \ldots, j$, we have $x_{i}+1 \notin Z\left(R_{i}\right)$ but $x_{i}+1 \in Z\left(R_{i}\right)$ for $i=j+1, \ldots, k$. We show that for $i=1, \ldots, k$, the edge $\{1, x\}$ does not belong to a maximal $\left(|R| / f_{i}\right)$-clique in $\tau(R)$ and this completes the proof. 
Let $\left\{y_{s} \mid s \in S\right\}$ be a set of elements of $R$ of maximal size which are adjacent to both 1 and $x$ and also to themselves. And, let $\left[a_{m}\right]$ denote the equivalence class of $Z\left(R_{m}\right)+a_{m}$. If $\left\{y_{s} \mid s \in S\right\} \cup\{x, 1\}$ forms a clique of maximal size $|R| / f_{i}$, then there must be $1 \leq m_{1}<m_{2}<\ldots<m_{q} \leq k$; $0 \leq q \leq k$ such that all $y_{s}$ 's belong to

$R_{1} \oplus \cdots \oplus R_{m_{1}-1} \oplus\left[a_{m_{1}}\right] \oplus R_{m_{1}+1} \oplus \cdots \oplus R_{m_{q}-1} \oplus\left[a_{m_{q}}\right] \oplus R_{m_{q}+1} \oplus \cdots \oplus R_{k}$.

The case $q=0$ is not possible. To the contrary, suppose that $q=0$. Consequently, we must have

$$
|R| / f_{i}= \begin{cases}k & k \text { is even } \\ k+1 & k \text { is odd, }\end{cases}
$$

for some $i=1, \ldots, k$. But, if $k>2$, then the inequality $k+1<3^{k-1}$ holds, and hence $|R| / f_{i}<3^{k-1} \leq|R| / f_{i}$, which is a contradiction. The case $k=2$ also implies a similar contradiction.

If a vertex $y=\left(y_{1}, \ldots, y_{k}\right)$ is adjacent to both 1 and $x$ in $\tau(R)$, then because each $f_{i} \geq 3$ and $\left|R_{j+1}\right|, \ldots,\left|R_{k}\right|$ are all odd, there is not an $i, 1 \leq$ $i \leq k$, such that $y_{i}+1$ and $y_{i}+x_{i} \in Z\left(R_{i}\right)$. Hence if $y=\left(y_{1}, \ldots, y_{k}\right)$ is adjacent to both 1 and $x$, then there must exist $s, t \in\{1, \ldots, k\}$ with $s \neq t$ such that $y_{s}+1 \in Z\left(R_{s}\right)$ and $y_{t}+x_{t} \in Z\left(R_{t}\right)$. Therefore, $q \neq 1$.

Now, suppose that $2 \leq q \leq k$. We must have $\left[a_{m_{p}}\right]=\left[-1_{m_{p}}\right]$ and $\left[a_{m_{v}}\right]=\left[-x_{m_{v}}\right]$ for some $v \neq p, 1 \leq p, v \leq k$, because otherwise the $y_{s}$ 's are not adjacent to 1 or $x$. Meanwhile, for some $t \in\{1, \ldots, q\}$, we must also have $m_{t} \leq j$, because otherwise the $y_{s}$ 's cannot be adjacent to themselves. Then, for each $i=1, \ldots, k$ we have $\left|\left\{y_{s} \mid s \in S\right\}\right|+2=\frac{|R|}{\prod_{i=1}^{q} f_{m_{i}}}+2 \neq \frac{|R|}{f_{i}}$. To the contrary, suppose that $\frac{|R|}{\prod_{i=1}^{q} f_{m_{i}}}+2=\frac{|R|}{f_{i}}$ for some $i=1, \ldots, k$. Let $n$ be the greatest integer such that $2^{n}|| R \mid$, i.e., $|R|=2^{n} r$ for some odd integer $r \neq 1$. Since $R_{m_{1}}, \ldots, R_{m_{t}}$ are local rings of even orders, it can be inferred that $\prod_{i=1}^{t} f_{m_{i}}=2^{g}$ for some positive integer $g, 2 \leq g \leq n$. Thus, we have

$$
\frac{2^{n} r}{2^{g} \prod_{i=t+1}^{q} f_{m_{i}}}+2=\frac{2^{n-g} r}{\prod_{i=t+1}^{q} f_{m_{i}}}+2=2\left(\frac{2^{n-g-1} r}{\prod_{i=t+1}^{q} f_{m_{i}}}+1\right)=\frac{2^{n} r}{f_{i}} .
$$

Hence,

$$
\frac{2^{n-g-1} r}{\prod_{i=t+1}^{q} f_{m_{i}}}+1=\frac{2^{n-1} r}{f_{i}} .
$$

If $n-g-1>0$, then the previous equality is impossible since the right hand side is always an even integer but the left hand side is odd. The case $n-g-1=0$ is not possible, because it means that one of the $f_{i}$ 's equals 2 , 
a contradiction to our assumption. The case $n-g-1=-1$ cannot satisfy the previous equality, because the left hand side is not an integer, while the right hand side is always an integer. Consequently, the case $2 \leq q \leq k$ is not possible either.

Therefore, in $\tau(R)$, the set $\left\{y_{s} \mid s \in S\right\} \cup\{x, 1\}$ cannot form a clique of maximal size $\frac{|R|}{f_{i}}$ for each $i, i=1, \ldots, k$. Hence $\tau(R) \nsucceq C(R)$.

Remark 5.3. The two conditions (a) and (b) in Theorem 5.2 are not distinct, i.e., they can both be true for a ring $R$.

\section{ACKNOWLEDGMENTS}

The authors owe a great debt to the referees who have carefully read an earlier version of this paper and made significant suggestions for improvement. We would like to express our deep appreciation for the referees' work.

\section{REFERENCES}

Akbari, S., Kiani, D., Mahammadi, F., Moradi, S. (2009). The total graph and regular graph of a commutative ring, J. Pure Appl. Algebra 213: 22242228 .

Akhtar, R., Bogges, M., Jackson-Henderson, T., Jiménez, I., Karpman, R., Kinzel, A., Pritikin, D. (2009). On the unitary Cayley graph of a finite ring, Electronic J. of Combinatorics 16: \#R117.

Anderson, D. F., Badawi, A. (2008). The total graph of a commutative ring, J. Algebra 217: 2706-2719.

Atiyah, M. F., Macdonald, I. G. (1969). Introduction to commutative algebra, Addison-Wesley Publishing Co.

Behzad, M. (1970). A Characterization of total graphs, Proceedings Amer. Math. Society 26(3): 383-389.

Beineke, L. W., Wilson, R. J. (2004). Topics in algebraic graph theory, Cambridge University Press.

Maimani, H. R., Wickham, C., Yassemi, S. Rings whose total graphs have genus at most one, Rocky Mountain J. Math.: to appear.

West, D. B. (2000). Introduction to graph theory, second edition, PrenticeHall. 